\title{
Future Home Exploration Based on Artificial Intelligence Tong $\mathrm{Li}^{*}$ \\ North China Electric Power University (Baoding), Hebei, China \\ *1151859092@qq.com
}

\begin{abstract}
As a new industry, smart home is at a critical point in the period of introduction and growth. The concept of market consumption has not yet been formed. However, with the further implementation of the promotion of the smart home market, consumers 'usage habits have been cultivated. The consumption potential of intelligent home market must be huge, the industry prospect is bright.
\end{abstract}

Keywords: Al; smart home; artificial intelligence; internet of things.

\section{Research Background}

With the increasing development of society and the improvement of people's living standards, people have higher requirements for the home environment. Service robots have successively entered the public view. Intelligent home is an indispensable part of the Internet of Things industry and is supported by national policies. The robot industry development plan also clearly set out the goals for the development of the robot industry. Among them, the annual sales revenue of service robots exceeds 30 billion yuan.

\section{Industry History}

As a new industry, smart home is at a critical point in the period of introduction and growth. The concept of market consumption has not yet been formed. However, with the further implementation of the promotion of the smart home market, consumers 'usage habits have been cultivated. The consumption potential of intelligent home market must be huge, the industry prospect is bright. Smart home has experienced nearly 12 years of development in China, from people's initial dream to today's real into our lives, has gone through a difficult process.

The development of smart home in China has gone through five stages, namely, budding period, pioneering period, lingering period, Fusion evolution period and outbreak period.

\section{Product Description}

The company's main products include smart home robots, recycling lockers and laser pianos.

\subsection{Intelligent Home Robot}

Intelligent Home Robot is a multi-functional home robot with practical functions. It integrates home service functions such as sweeping the floor and delivering meals. On this basis, it organically integrates home with the booming smart interconnection theme. At the same time, it has the functions of intelligent home network such as voice interaction and infrared remote control.

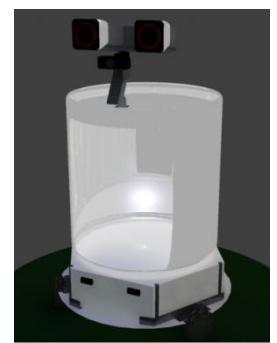

Figure 1. Robot structure physical map

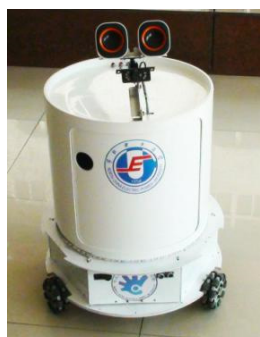

Figure 2. Robot overall effect map 


\subsection{Circular Lockers.}

Circular lockers are smart vertical lockers that can be moved. The height of the wardrobe in the family is generally about 0.5 meters away from the roof or high with the roof, resulting in a decline in space utilization and inconvenience in accessing items at high altitudes. This product cabinet can move independently according to a certain route, facilitate access to items, improve space utilization, and give users an intelligent experience.

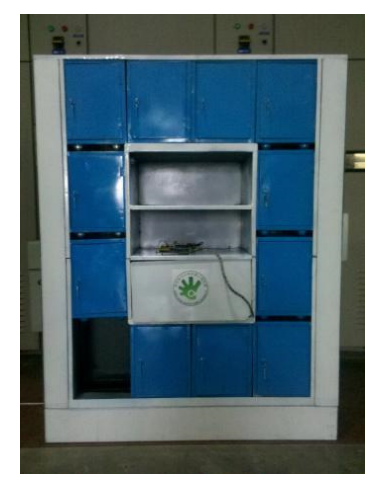

Figure 3. Physical Chart of Circular Lockers

\subsection{The Laser Piano.}

The laser piano, which uses laser laser to replace traditional piano keys, is a research direction of a new type of smart keyboard. Users can play music through virtual performances at laser points, which is used for incomparable dimensions and price advantages compared to traditional electronic pianos.

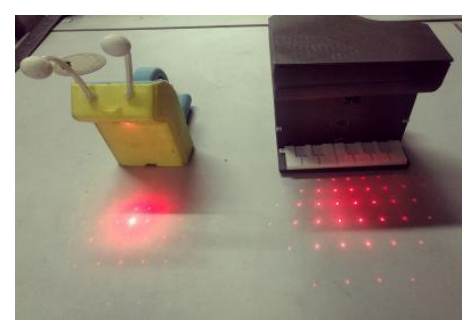

Figure 4. First and second-generation laser piano models

\section{Product Innovation Points}

\subsection{Useful and Easy to Use.}

The basic goal of intelligent home is to provide people with a comfortable, safe, convenient and efficient living environment. Our products are practical as the core, discard those flashy, can only be used as a function of furnishings, the product is practical, easy to use and Humanized.

\subsection{High Reliability.}

Every intelligent subsystem of our products can run 24 hours a day, and the safety, reliability and fault tolerance of household robots and lockers are strong. Domestic robots have the ability to cope with complex environmental changes. Ultrasonic sensors are installed in front of the robots. Ultrasound can detect the distance of objects in front of them. Therefore, according to this distance, it is possible to determine whether there are obstacles in front of them, to automatically avoid obstacles, and to ensure users. Safety.

\subsection{The Standards are Strict.}

The design of the product plan is strictly in accordance with the standards, ensuring the expansibility and expansibility of the system. The standard TCP/IP protocol network technology is 
used for system transmission to ensure that the systems between different producers are compatible and interconnected. The system of the product interconnects with the third party-controlled equipment that will continue to develop in the future. In order to facilitate the later function expansion, simple, reliable, convenient and economical.

\section{4 Convenient Installation and Maintenance.}

Home intelligence has a remarkable feature, that is, installation, commissioning and maintenance of a very large workload, a large amount of human and material resources are required to invest, become a bottleneck restricting the development of the industry. In response to this problem, our products can be avoided. Robots do not need to wire. When designing circular lockers, they consider the convenience of installation and maintenance. The laser piano is a minimalist power supply system, bringing users and manufacturers the greatest convenience.

\section{Opportunities and Challenges}

Opportunity: There are a large number of domestic companies engaged in a variety of smart home products and R\&D, especially in the Beijing-Tianjin-Hebei region, but there are fewer scale enterprises, there is no certain market scale and brand effect, and the user experience is poor. Our products are safe and reliable enough to be innovative and ornamental, will bring consumers a good physical inspection, and there are few multi-functional intelligent home robot products on the market, with fewer competitors.

Challenge: At present, traditional home still occupies most of the market share of home market, smart home due to high prices, resulting in a small audience, low market share. In the era of "Internet $+"$, technology upgrading is fast, and companies need to continuously develop and maintain technological advantages.

\section{Summary}

In the current market, similar to our company's flagship products are rare, and the number of products with relevant authoritative patent guarantees is even more insignificant. And my company is using this as a basis to face the market competition, establish a good image, so that users can rest assured. At the same time, our company will carry out related promotion, snap up and other measures to attract customers. In the previous year's sales, our company will regularly carry out user feedback, to the users of various issues to adjust the product, constantly innovative product technology. Do your best to meet all the needs of users, to win the praise of users constantly. And in the market, the company should always be ready to deal with the issues, regular meetings, to guard against risks, prevention.

\section{References}

[1]. Xieyonghua, Peilonglong, Wu Tong. Application of the Internet of Things in Smart Home [ J] Instrument Technology, 2015(3): 46-49.

[2]. Chen Fei. On Internet of Things Intelligent Home Development [ J] Science and technology Outlook, 2016, 26(8): 147-148.

[3]. Zhouwu. Based on the Internet of Things Intelligent Home Development Analysis[J] Information technology and informatization. 2015(02).

[4]. Wu Sinan. Design and Implementation of Intelligent Home Control System Based on Internet of Things [ D] Yangzhou University 2016. 